\title{
Endogenous development of Hemolivia mauritanica (Apicomplexa: Adeleina: Haemogregarinidae) in the marginated tortoise Testudo marginata (Reptilia: Testudinidae): evidence from experimental infection
}

\author{
Pavel Široký ${ }^{1}$, Martin Kamler ${ }^{2}$, Fredric L. Frye ${ }^{3}$, Petr Fictum ${ }^{4}$ and David Modrý $\dot{y}^{2,5}$ \\ ${ }^{1}$ Department of Biology and Wildlife Diseases, ${ }^{2}$ Department of Parasitology and ${ }^{4}$ Department of Pathology, University of \\ Veterinary and Pharmaceutical Sciences, Palackého 1-3, 61242 Brno, Czech Republic; \\ ${ }^{3}$ La Primavera Farm, 33422 Highway 128, Cloverdale, California 95425-9428, USA; \\ ${ }^{5}$ Institute of Parasitology, Biology Centre, Academy of Sciences of the Czech Republic, Branišovská 31, 37005 České \\ Budějovice, Czech Republic
}

Key words: Apicomplexa, Hemolivia mauritanica, Testudo, merogony, cystogony, tick-born disease, life cycle

\begin{abstract}
Six young tortoises Testudo marginata Schoepff, 1792 were experimentally infected with Hemolivia mauritanica (Sergent et Sergent, 1904). The prepatent period ranged from 6 to 8 weeks. Young, smaller, club-like forms $(6-9 \times 3-6 \mu \mathrm{m})$ of gametocytes appeared in the peripheral blood first, whereas mature, elongated, cylindrical forms $(9-12 \times 5-7 \mu \mathrm{m})$ were detected after 1-2 weeks and predominated during later patency. Three of the infected tortoises were euthanized and dissected to study the endogenous stages. Meronts occurred in the cells of the reticulo-endothelial system and in the erythrocytes; these were observed mostly in parenchymatous organs. Mature forms measured $14.2 \times 9.3 \mu \mathrm{m}$ and contained 7-12 merozoites. Cysts with two (exceptionally one) cystozoites were also found predominantly in parenchymatous organs and measured $14.8 \times 7.9 \mu \mathrm{m}$. Pathological changes attributable to Hemolivia were mild and limited to liver and kidneys. The role of individual developmental stages of haemogregarines is discussed with respect to evolution of heteroxenous life cycle and long-term persistence of parasites in their intermediate hosts.
\end{abstract}

The genus Hemolivia Petit, Landau, Baccam et Lainson, 1990 is defined as a haemogregarine of ectothermic vertebrates with presence of erythrocytic gamogony, and erythrocytic and extra-erythrocytic merogony and cystogony. Sporogony consisting of two phases occurs within gut epithelial cells of the definitive hosts, ixodid ticks, which serve as vectors. In the first phase, conjugation and fertilisation of macrogametes by microgametes occurs, followed by the formation of oocysts, which contain the motile sporokinetes (without formation of sporocysts). In the second phase, sporokinetes released from oocysts, penetrate new cells to produce sporocysts, later containing sporozoites, infective to intermediate vertebrate host $(\mathrm{IH})$. There is no evidence of transovarian transmission within ticks (Petit et al. 1990).

The type species of the genus, Hemolivia stellata Petit, Landau, Baccam et Lainson, 1990, infects toads Bufo marinus (Linnaeus, 1758) and ticks Amblyomma rotundatum Koch, 1844 . The genus currently contains two other species, H. mariae Smallridge et Paperna, 1997 affecting lizards Tiliqua rugosa (Gray, 1825) and ticks Amblyomma limbatum Neumann, 1899, and $H$. mauritanica (Sergent et Sergent, 1904) from the Palaearctic tortoises of the genus Testudo Linnaeus, 1758 and ticks Hyalomma aegyptium (Linnaeus, 1758) (Sergent and Sergent 1904, Petit et al. 1990, Smallridge and Paperna 1997).

Hemolivia mauritanica was originally described as Haemogregarina mauritanica, from an Algerian specimen of the spur-thighed tortoise Testudo graeca Linnaeus, 1758 (Sergent and Sergent 1904). Laveran and Nègre (1905) and Brumpt (1938) provided a description of its life cycle, developmental stages, and oral transmission via tortoises feeding on infected ticks. Michel (1973) on the basis of characters of the parasite's sporogony and merogony transferred it to the genus Hepatozoon Miller, 1908. Finally, Landau and Paperna (1997), after the reexamination of Brumpt's original material, revised the taxonomic state of the parasite, which is currently considered to be a member of the genus Hemolivia. Recently, we have found $H$. mauritanica in blood smears from wild, as well as from longterm captive, marginated tortoises Testudo marginata Schoepff, 1792 from Greece (Široký et al. 2004, 2005).

Despite the fact that more than hundred years have passed since $H$. mauritanica was first described, its life cycle, natural means of infection and mechanisms for long-term persistence in the host remain unknown. The goal of our study was to elucidate the endogenous development and morphology of the developmental stages of $H$. mauritanica in its newly recognized host, T. marginata.

Address for correspondence: P. Široký, Department of Biology and Wildlife Diseases, University of Veterinary and Pharmaceutical Sciences, Palackého 1-3, 61242 Brno, Czech Republic. Phone: ++420 541562 635; E-mail: sirokyp@vfu.cz 


\section{MATERIALS AND METHODS}

Adult Hyalomma aegyptium from a laboratory breeding colony were allowed to feed on a haemogregarine-positive $T$. marginata. After their detachment, the ticks were dissected and examined for the presence of Hemolivia sporocysts. One infected tick was gently force-fed to each of six two-year-old haemogregarine-negative $T$. marginata. Blood was collected by puncture of the dorsal coccygeal vein once weekly. Blood smears were air-dried, fixed in absolute methanol and stained with Giemsa (diluted 1:10 in buffered water, $\mathrm{pH}$ 7) for 20 minutes, and examined with an Olympus Provis AX-70 microscope at $\times 1,000$ magnification. During the prepatent period, the presence and morphology of both premature and mature gametocytes within the red blood cells (RBCs) were recorded. The size of infected RBCs was compared with those of uninfected cells. We counted only mature forms of erythrocytes; young polychromatophilic forms of RBCs were excluded from this analysis. Intensity of parasitaemia was counted as the number of infected RBCs for 10,000 cells.

Three tortoises having the highest level of parasitaemia were euthanized 10 (No. 1), 14 (No. 2) and 17 weeks (No. 3) post-infection, by overdosing with the barbiturate (Thiopental ICN), followed by decapitation. Lung, liver, kidney, spleen, brain, stomach, gut, heart, and skeletal muscle were sampled. Each organ was examined immediately as a fresh squash preparation and a portion was fixed in $10 \%$ buffered form- aldehyde for histopathological examination. After the dehydration in a graded ethanol series, samples were embedded in paraffin, cut to $4 \mu \mathrm{m}$ thickness and stained by standard haematoxylin-eosin procedure.

\section{RESULTS}

All six juveniles of $T$. marginata became infected with a prepatent period ranging from 6 to 8 weeks. Young forms of gametocytes (or merozoites sensu Smallridge and Bull 2001) appeared in the peripheral blood in the beginning of patent period; the mature forms appeared after 1-2 weeks and predominated later during the patency. In tortoise No. 1, 21.3\% of the RBCs were infected 9 weeks post-infection, while in remaining tortoises (Nos. 2-5) parasitaemias of 5.9, 4.2, 1 and $0.8 \%$ infected RBCs respectively were recorded. In tortoise No. 6, only four infected RBCs per 10,000 cells were detected.

\section{Developmental stages}

Gametocytes in the peripheral blood

Figs. 1-5

Gametocytes at different developmental stages were located solely in erythrocytes. The premature gametocytes were first small, club-like, 6-9 × 3-6 $\mu \mathrm{m}(\mathrm{n}=11$; Fig. 1). Larger forms became more oblong, and meas-

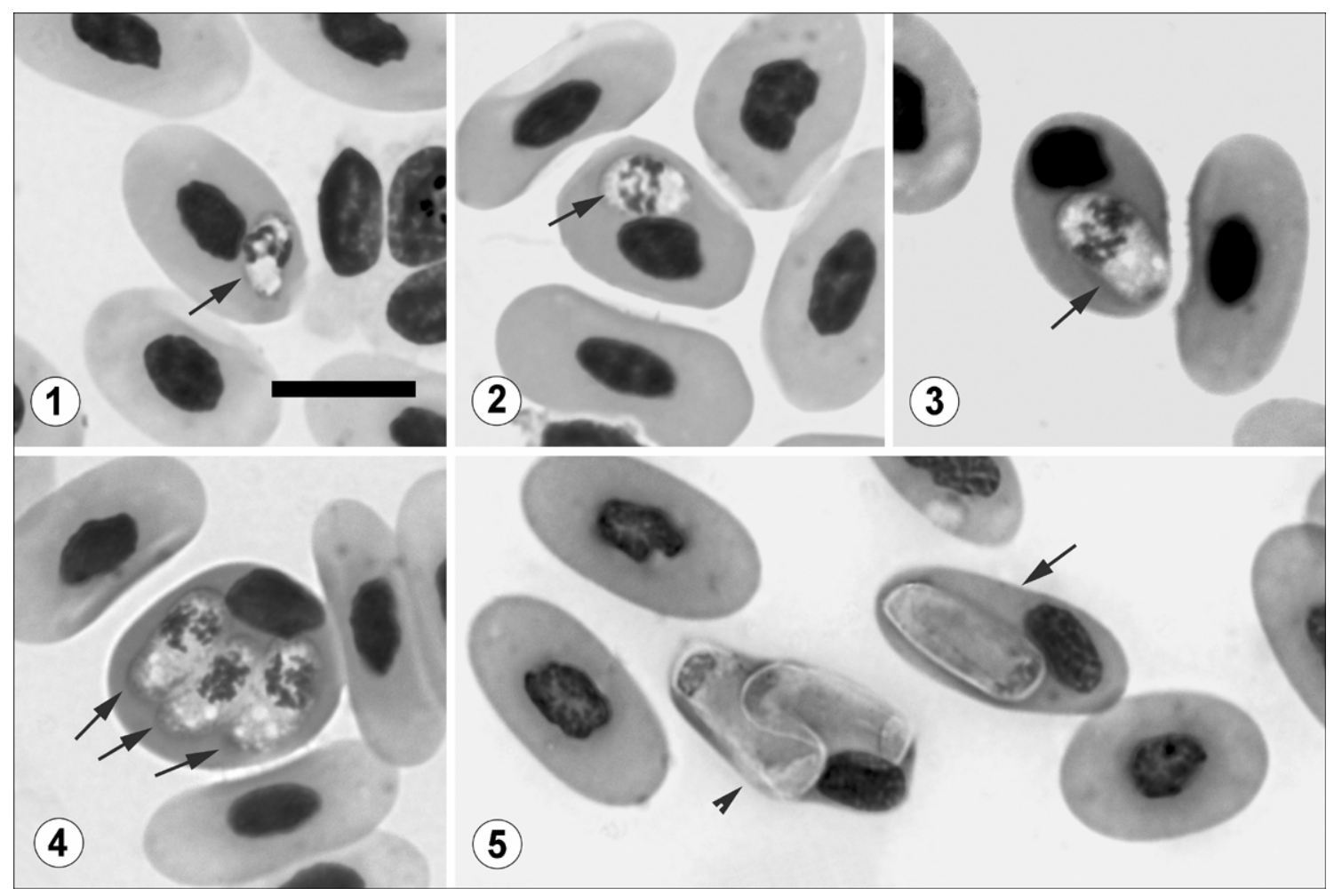

Figs. 1-5. Hemolivia mauritanica, development of gametocytes within the erythrocytes of peripheral blood of Testudo marginata, tortoise No. 4. Fig. 1. Youngest club-like form marked by arrow, 49 days post-infection (DPI). Fig. 2. Premature oblong form of gametocyte (arrow), 49 DPI. Fig. 3. Premature gametocyte (arrow) with nucleus located sub-centrally, 49 DPI. Fig. 4. Erythrocyte infected by three premature gametocytes (arrows) simultaneously, 56 DPI. Fig. 5. Mature gametocyte with stainresistant capsule and nucleus in polar position (arrow) and erythrocyte infected by two gametocytes (arrowhead), 165 DPI. Scale bar $=10 \mu \mathrm{m}$; all figures in the same scale. 


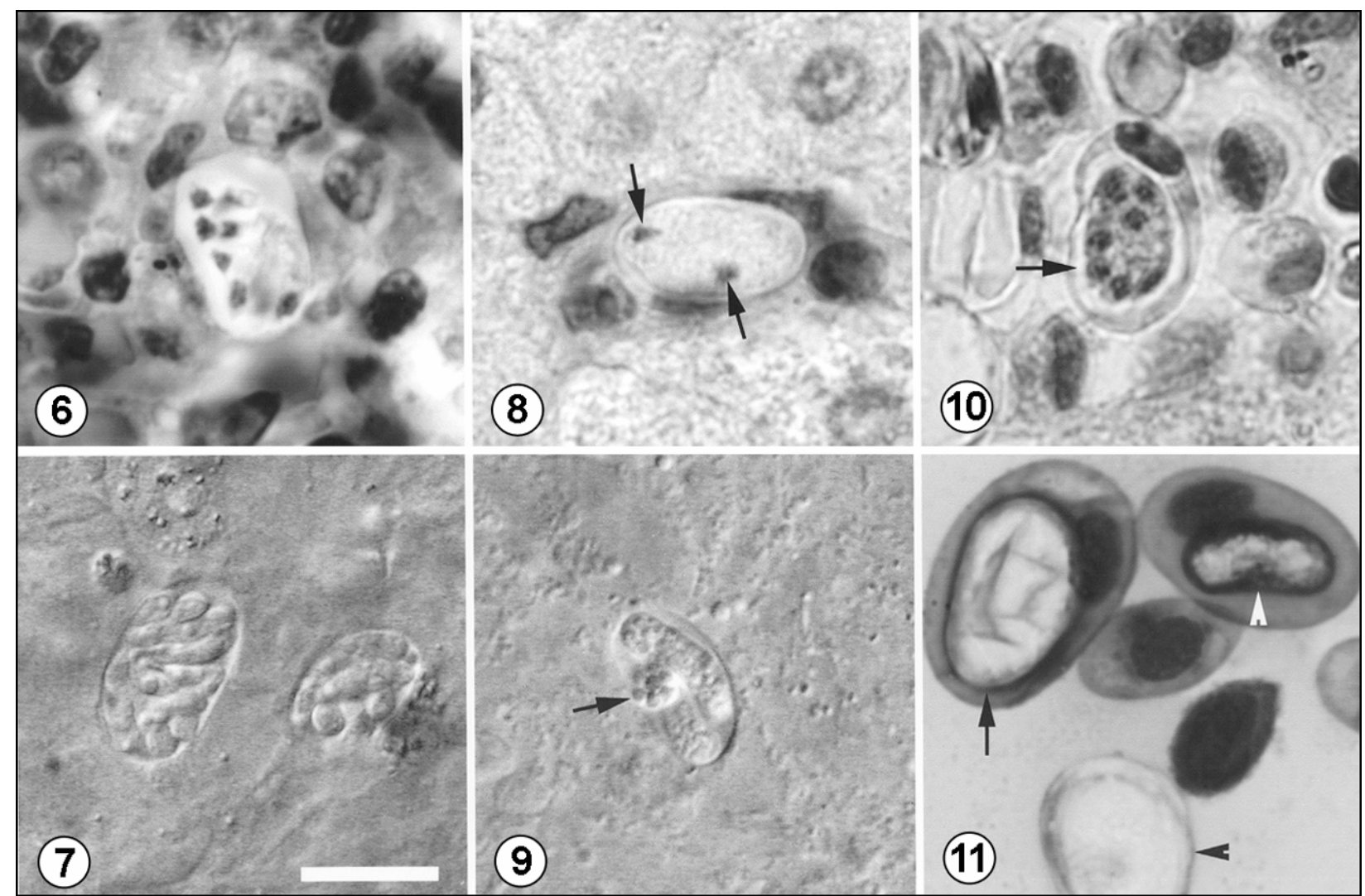

Figs. 6-11. Meronts and cysts of Hemolivia mauritanica in histological sections (Figs. 6, 8, 10 - haematoxylin-eosin staining), unstained compressed slides (Figs. 7, 9 - Nomarski interference contrast), and blood smear stained with Giemsa (Fig. 11). Fig. 6. Meront in the spleen, tortoise No. 1, 70 DPI. Fig. 7. Two meronts in the liver of tortoise No. 1, 70 DPI. Fig. 8. Dizoic cyst in the liver, nuclei of cystozoites marked by arrows, tortoise No. 1, 70 DPI. Fig. 9. Dizoic cyst in the spleen, cyst residual body marked by an arrow, tortoise No. 3, 119 DPI. Fig. 10. Intraerythrocytic merogony, meront marked by an arrow, tortoise No. 1, 70 DPI. Fig. 11. Meront within the erythrocyte (arrow), free meront (black arrowhead) probably after destruction of erythrocyte, and premature gametocyte (white arrowhead), tortoise No. 1, 70 DPI. Scale bar $=10 \mu \mathrm{m}$; all figures in the same scale.

ured 7-10 × 4-6 $\mu \mathrm{m}(\mathrm{n}=12$; Figs. $2-4)$, with their nucleus located sub-centrally. Mature gametocytes were elongated, oval to cylindrical, sometimes bean-shaped 9-12 × 5-7 $\mu \mathrm{m}(\mathrm{n}=22)$; they developed a stainresistant capsule and nucleus located in a polar position (Fig. 5). Gametocytes affected the shape of infected RBCs to some degree. Uninfected erythrocytes measured $19.4(17-24) \times 10.7(9-15) \mu \mathrm{m}$, shape index (SI, length/width) of $1.83(1.38-2.33)(\mathrm{n}=30)$, whereas infected RBCs were shorter in average $17.1(12-21) \times$ $10.6(8-15) \mu \mathrm{m}$, SI $1.63(1.07-2.11)(\mathrm{n}=30)$ (MannWhitney test; length: $Z=4.12, \mathrm{p}<<0.01$; width: $\mathrm{Z}=$ $0.76, p=0.45$; SI: $Z=-13.08, p<<0.01)$.

\section{Meronts}

Figs. 6, 7, 10, 11

Merogony occurred in the cells of reticuloendothelial system (Figs. 6, 7) and in the erythrocytes (Figs. 10,11) primarily in the spleen, liver, kidneys and lungs. Meronts at different developmental stages were always observed within blood vessels and sinusoids. They were never seen in the parenchyma of internal organs. Primary and secondary meronts were not distinguished. The nuclei of premature meronts were arranged around the peripheral margin of the cytoplasm, and measured $11.3(10-12) \times 8.3(6-9) \mu \mathrm{m}(\mathrm{n}=13)$. Mature meronts measured $14.2(13-17) \times 9.3(7-11) \mu \mathrm{m}(\mathrm{n}=$ 17) and contained 7-12 elongate merozoites, usually arranged in parallel within the long axis of meront.

Cysts

Figs. 8, 9

These stages were found in the same locations as meronts, particularly in parenchymatous organs such as liver, spleen and kidneys. Interestingly, within the organs of specimen No. 3 examined 17 weeks postinfection the cystic stage predominated over the meronts. The cysts contained mostly two, exceptionally one, elongate, cucumber-shaped cystozoites, having a spongiform appearance in stained histological sections, whereas in fresh squash films they appeared finely granular with circular nucleus located sub-centrally. Spherical granules formed the residual body of the cyst (Fig. 9). Cysts were elongate, ellipsoidal to beanshaped, measuring $14.8(12-18) \times 7.9(6-10) \mu \mathrm{m}$ in dizoic form $(\mathrm{n}=26)$ and $13 \times 7(6-8) \mu \mathrm{m}$ in case of rare monozoic cysts $(n=3)$. 


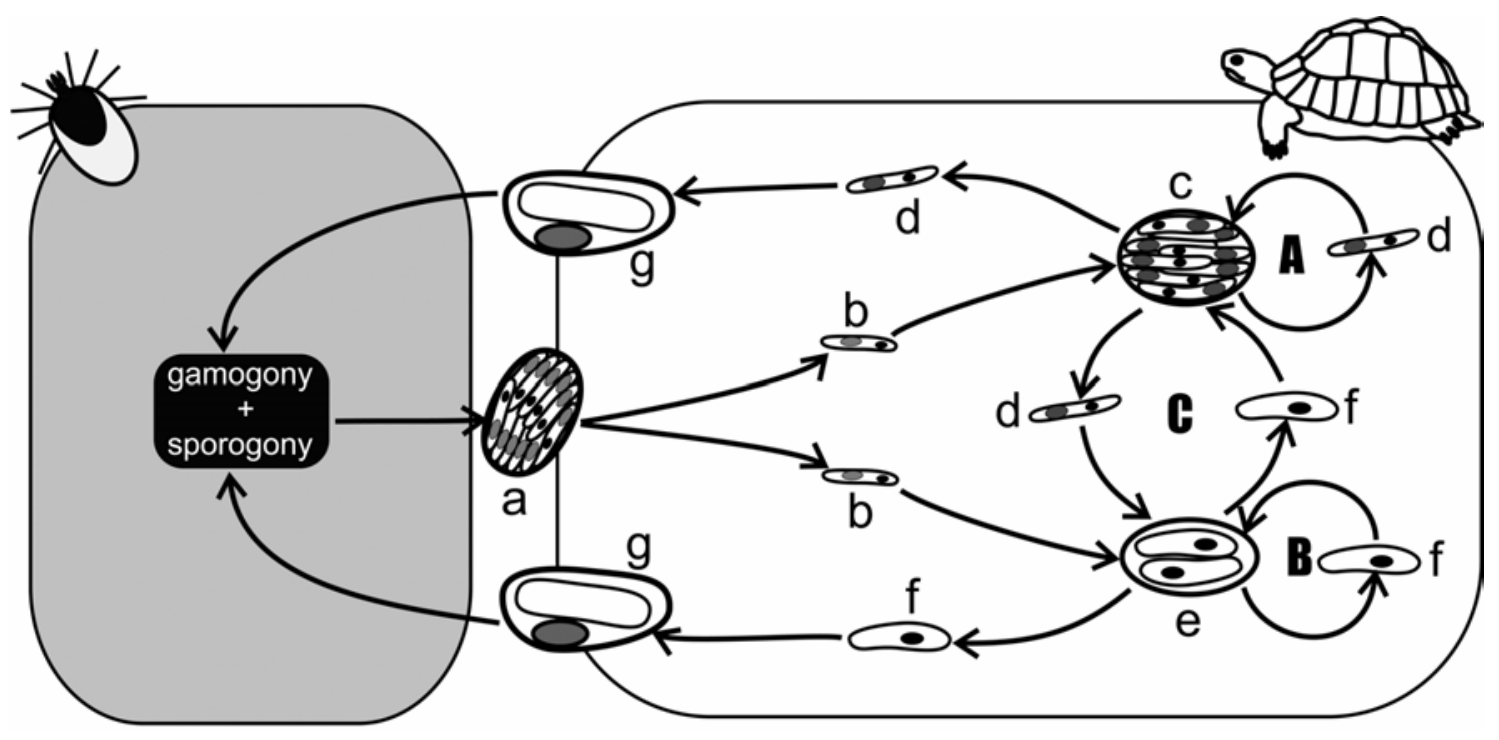

Fig. 12. Development of Hemolivia mauritanica in Testudo marginata shown in an open square area (development in the tick is not discussed, shown as shaded area). Sporocysts (a) located in tick are ingested by tortoise. Sporozoites (b) enter cells of the reticulo-endothelial system or erythrocytes, where they transform either to meronts (c) containing merozoites (d) or to cysts (e) with cystozoites (f). Gametocytes (g) develop in erythrocytes invaded either by merozoites or cystozoites. Three ways of longterm maintaining of infection are hypothesized: (A) cyclic merogony, (B) cyclic cystogony, and (C) initiation of merogony by cystozoites or conversely, initiation of cystogony by merozoites.

\section{Pathological changes}

Mild pathological changes attributable to Hemolivia were only observed in histological sections from the tortoise which exhibited the highest parasitaemia. These mild alterations were confined to the kidneys and liver and consisted of mild focal to multifocal lower nephron nephrosis and hepatocellular hydropic degeneration. Developmental stages of Hemolivia were observed within hepatic sinusoids; however, the hepatic parenchyma remained normal. The balance of the parenchymatous organs from all tortoises appeared normal, regardless of the degree of intraerythrocytic parasitism observed.

\section{DISCUSSION}

All experimentally inoculated tortoises became infected with Hemolivia mauritanica, and displayed a prepatent period range comparable with that observed in other Hemolivia species (Petit et al. 2000, Smallridge and Bull 2001). Except for the absence of so-called primary merozoites (sensu Smallridge and Bull 2001), the occurrence and morphology of other intraerythrocytic forms correspond to those described by Smallridge and Bull (2001) for H. mariae in Australian skinks. However, we observed the intraerythrocytic meronts only in the blood smears collected from the tortoise with the highest parasitaemia.

Among the haemogregarines parasitizing ectothermic hosts, the genus Hepatozoon has been the most frequently studied. The majority of authors reported on two types of meronts in Hepatozoon, usually called macro- and micromeronts, or $\mathrm{Y}$ meronts and $\mathrm{X}$ meronts, respectively (i.a. Smith 1996, Telford et al. 2001, 2002, Paperna et al. 2002). So far, no species of Hemolivia have been reported to share this trait (Michel 1973, Petit et al. 1990, Paperna and Smallridge 2001). Although we found meronts differing by numbers of merozoites or by size, we were unable to distinguish two distinct groups. Rather, they displayed a continuous range, with the extremes resembling the so-called macro- and micromeronts. Compared to Michel (1973), we observed both premature and mature meronts smaller in average, and containing fewer merozoites.

The role of cystic stages in the life cycle of haemogregarines is enigmatic. Landau et al. (1972) first described the presence of cysts in the intermediate host of Hepatozoon domerguei. Since that time, the cystic stages have been recorded in many Hepatozoon species (e.g. Paperna et al. 2002, Lainson et al. 2003, Paperna and Lainson 2004) and also in members of the genus Hemolivia (Michel 1973, Petit et al. 1990). Michel (1973) recorded cysts containing mostly two, but rarely also four or six cystozoites in Hemolivia mauritanica. The majority of cysts we observed were dizoic; monozoic forms were rare. Neither tetrazoic nor hexazoic forms were seen. The cysts we observed, particularly the monozoic forms, were smaller and more elongated than those reported by Michel (1973) in Testudo graeca.

Landau et al. (1972) suggested that the cystic stages were responsible for transmission of Hepatozoon between lizards (first IH) and a saurophagous snake (second IH). Smith (1996) concluded that many Hepatozoon species in snakes undergo a three-host life cycle, where 
insectivorous frogs or lizards serve as the first $\mathrm{IH}$, a snake predating on them is the second $\mathrm{IH}$, and mosquitoes as definitive hosts that can feed on all - frogs (or lizards) and snakes. Subsequently, Smith and Desser (1998) described the life cycle of Hepatozoon sipedon with cystogony in frogs Rana pipiens, preceding the merogony in snakes Nerodia sipedon that were fed the liver of these frogs. Petit et al. (1990) suggested a complex role of cysts in oral transmission of Hemolivia stellata to toad, Bufo marinus. Such a "transmission hypothesis" fits well to haemogregarines with predatory intermediate hosts. However, the intermediate hosts of $H$. mauritanica are herbivorous tortoises. Thus, cysts of $H$. mauritanica either have some other function (see below) or represent an evolutionary relic stage inherited from a common ancestor. A second explanation suggests the transition from a three-host to a two-host life cycle. Recent studies focusing on the phylogeny of blood apicomplexans suggested their evolution from monoxenous apicomplexans of invertebrates (Barta 1989, Kopečná et al. 2006). Then, the ancient origin of two-host life cycle of $H$. mauritanica is more likely than secondary simplification from a more complicated three-host development.

With respect to the studies mentioned above, possible ways of endogenous development of $H$. mauritanica in tortoises are hypothesized in Fig. 12. Cystogony could serve as a parallel path to merogony and can represent an adaptation, responsible for long-term (perhaps even life-long) persistence of the infection. Our previous studies documented that $H$. mauritanica gametocytes can occur in a tortoises' circulating blood for at least 12 years (Šroký et al., unpubl.). The lifespan of reptilian erythrocytes is definitely shorter (e.g. Davies and Johnston 2000) and gametocytes are considered to be the final stage of development in the vertebrate host. Therefore, long-term parasitaemia must be maintained somehow. The permanent presence of gametocytes can be explained several ways: (i) cyclic merogony, (ii) cyclic cystogony, or (iii) by consecutive release of dormant cystozoites or merozoites. Further, it is possible (iv) that dormant cystozoites can initiate another cycle of merogony after their activation.

Endogenous extra-erythrocytic development of haemosporidia is known to be responsible for inducing considerable pathology, both in birds and mammals. In contrast, haemogregarines are not considered to be particularly pathogenic (Desser 1993) and few studies deal with pathology caused by them. Reported changes include the alteration of erythrocytes' morphology and their overall shape, hypertrophy of cytoplasm or pycnotic and dislocated nuclei without pronounced clinical effect (Ball et al. 1969, Allison and Desser 1981, Siddall and Desser 1993). Meront-associated inflammatory lesions resulting in clinical disease were described by Wozniak et al. (1998). Pathology was observed in unnatural lizard hosts infected experimentally with Hepatozoon species that was transferred to them from naturally infected snakes (Wozniak and Telford 1991, Wozniak et al. 1996). In our study, only mild pathological changes were recorded in the kidneys and liver of the tortoise manifesting the highest parasitaemia. Hemolivia mauritanica infection does not appear to represent a serious threat for captive or wild tortoises of the genus Testudo.

Acknowledgements. This study was, in part, supported by the grant from the University of Veterinary and Pharmaceutical Sciences, Brno, project IGA VFU 2/2004/FVHE and by the grants from the Grant Agency of the Czech Republic, No. $524 / 03 /$ D 104 and No. 524/03/H133. We are grateful to Jan Erhart for help with rearing the Hyalomma ticks and to František Tichý and Miša Škorič for the assistance with interpretation of histological findings.

\section{REFERENCES}

ALLISON B., DESSER S.S. 1981: Developmental stages of Hepatozoon lygosomarum (Doré 1919) comb. n. (Protozoa: Haemogregarinidae), a parasite of a New Zealand skink, Leiolopisma nigriplantare. J. Parasitol. 67: 852-858.

Ball G.H., ChaO J., Telford S.R. JR. 1969: Hepatozoon fusifex sp. n., a hemogregarine from Boa constrictor producing marked morphological changes in infected erythrocytes. J. Parasitol. 55: 800-813.

BARTA J.R. 1989: Phylogenetic analysis of the class Sporozoea (phylum Apicomplexa Levine, 1970): evidence for independent evolution of heteroxenous life cycles. J. Parasitol. 75: 198-206.

BRUMPT E. 1938: Formes évolutives d'Homogregarina mauritanica chez la tique Hyalomma syriacum. Ann. Parasitol. 16 350-361.

DAVIES A.J., Johnston M.R.L. 2000: The biology of some intraerythrocytic parasites of fishes, amphibia and reptiles. Adv. Parasitol. 25: 1-107.

DESSER S.S. 1993: The Haemogregarinidae and Lankesterellidae In: J.P. Kreier and J.R. Baker (Eds.), Parasitic Protozoa. Second Edition, Vol. 4. Academic Press, New York, pp. 247272 .
KOPEČNÁ J., JIRKŮ M., OBORNÍK M., TOKAREV Y.S., LUKEŠ J., MODRÝ D. 2006: Phylogenetic analysis of coccidian parasites from invertebrates: search for missing links. Protist 157: 173183.

LAINSON R., DE SOUZA M.C., FrANCO C.M. 2003: Haematozoan parasites of the lizard Ameiva ameiva (Teiidae) from Amazonian Brazil: a preliminary note. Mem. Inst. Oswaldo Cruz 98: 1067-1070.

LANDAU I., Michel J.C., ChABAUd A.G. 1972: Cycle biologique d'Hepatozoon domerguei; discussion sur les caractères fundamentaux d'un cycle de coccidie. Z. Parasitenkd. 38: 250270.

LANDAU I., PAPERnA I. 1997: The assignment of Hepatozoon mauritanicum, a tick-transmitted parasite of tortoise, to the genus Hemolivia. Parasite 4: 365-367.

LAVERAN I., NĖGRE L. 1905: Sur un protozoaire parasite de Hyalomma aegyptium. C. R. Soc. Biol. 57: 964-966.

Michel J.-C. 1973: Hepatozoon mauritanicum (Et. et Ed. Sergent, 1904) n. comb., parasite de Testudo graeca: redescription de la sporogonie chez Hyalomma aegyptium et de la schizogonie tissulaire d'aprés le matériel d' E. Brumpt. Ann. Parasitol. (Paris) 48: 11-21. 
PAPERnA I., Kremer-MeCABell T., FinKelman S. 2002: Hepatozoon kisrae n. sp. infecting the lizard Agama stellio is transmitted by the tick Hyalomma cf. aegyptium. Parasite 9: $17-27$.

PAPERNA I., LAINSON R. 2004: Hepatozoon cf. terzii (Sambon \& Seligman, 1907) infection in the snake Boa constrictor constrictor from north Brazil: transmission to the mosquito Culex quinquefasciatus and the lizard Tropidurus torquatus. Parasite 11: 175-181.

PAPERNA I., SMALLRIDGE C.J. 2001: Ultrastructure of developmental stages of Hemolivia mariae (Apicomplexa: Haemogregarinidae), natural parasite of the Australian sleepy lizard, in experimentally infected deviant hosts. Folia Parasitol. 48: 255-262.

PetiT G., LANDAu I., BaCCAM D., Lainson R. 1990: Description et cycle biologique d'Hemolivia stellata n. g., n. sp., hémogrégarine de crapauds brésiliens. Ann. Parasitol. Hum. Comp. 65: 3-15.

SERGENT ED., SERGENT ET. 1904: Sur une hémogrégarine, parasite de Testudo mauritanica. C. R. Soc. Biol. 56: 130-131.

SIDDALL M.E., DeSSER S.S. 1993: Cytopathological changes induced by Haemogregarina myoxocephali in its fish host and leech vector. J. Parasitol. 79: 297-301.

ŠIROKÝ P., KAMLER M., MODRÝ D. 2004: Long-term occurrence of Hemolivia cf. mauritanica (Apicomplexa: Adeleina: Haemogregarinidae) in captive Testudo marginata (Reptilia: Testudinidae): evidence for cyclic merogony? J. Parasitol. 90: 1391-1393.

ŠIROKÝ P., KAMLER M., MODRÝ D. 2005: Prevalence of Hemolivia mauritanica (Apicomplexa: Adeleina: Haemogregarinidae) in natural populations of tortoises of the genus Testudo in the east Mediterranean region. Folia Parasitol. 52: 359-361.

SMALLRIDGE C.J., BULL C.M. 2001: Infection dynamics of Hemolivia mariae in the sleepy lizard Tiliqua rugosa. Parasitol. Res. 87: 657-661.
SMitH T.G. 1996: The genus Hepatozoon (Apicomplexa: Adeleina). J. Parasitol. 82: 565-585.

SMITH T.G., DESSER S.S. 1998: Ultrastructural features of cystic and merogonic stages of Hepatozoon sipedon (Apicomplexa: Adeleorina) in northern leopard frogs (Rana pipiens) and northern water snakes (Nerodia sipedon) from Ontario, Canada. J. Eukaryot. Microbiol. 45: 419-425.

SMITH T.G., KIM B., HONG H., DESSER S.S. 2000: Intraerythrocytic development of species of Hepatozoon infecting ranid frogs: evidence for convergence of life cycle characteristics among apicomplexans. J. Parasitol. 86: 451-458.

TELFORD S.R. JR., BUTLER J.F., TELFORD R.S. 2002: Hepatozoon species (Apicomplexa: Hepatozoidae) of the corn snake, Elaphe guttata (Serpentes: Colubridae) and the pigmy rattlesnake, Sistrurus miliarius barbouri (Serpentes: Viperidae) in south Florida. J. Parasitol. 88: 778-782.

TELFORD S.R. JR., WOZNIAK E.J., BUTLER J.F. 2001: Haemogregarine specificity in two communities of Florida snakes, with descriptions of six new species of Hepatozoon (Apicomplexa: Hepatozoidae) and a possible species of Haemogregarina (Apicomplexa: Haemogregarinidae). J. Parasitol. 87: 890905.

WOZNIAK E.J., KAZACOS K.R., TELFORD S.R. JR., MCLAUGHLIN G.L. 1996: Characterization of the clinical and anatomical pathological changes associated with Hepatozoon mocassini infections in unnatural reptilian hosts. Int. J. Parasitol. 26: 141-146.

WOZNIAK E.J., TELFORD S.R. Jr. 1991: The fate of Hepatozoon species naturally infecting Florida black racers and watersnakes in potential mosquito and soft tick vectors, and histological evidence of pathogenicity in unnatural host species. Int. J. Parasitol. 21: 511-516.

WOZNIAK E.J., TELFORD S.R. JR., DENARDO D.F., MCLAUGHLIN G.L., BUTLER J.F. 1998: Granulomatous hepatitis associated with Hepatozoon sp. meronts in a southern water snake (Nerodia fasciata pictiventris). J. Zoo Wildl. Med. 29: 68-71.

Accepted 1 February 2007 